\title{
Predominant neurological phenotype in a Hungarian family with two novel mutations in the XPA gene-case series
}

\author{
Dénes Zádori ${ }^{1}$ • László Szpisjak ${ }^{1}$ • István Balázs Németh ${ }^{2}$ - Zita Reisz ${ }^{3}$. Gabor G. Kovacs ${ }^{4}$. Noémi Szépfalusi ${ }^{1}$. \\ Viola Luca Németh ${ }^{1}$. Zoltán Maróti ${ }^{5}$. Edit Tóth-Molnár ${ }^{6}$. Judit Oláh ${ }^{2}$ • László Vécsei ${ }^{17,8}$ • Péter Klivényi ${ }^{1}$. \\ Tibor Kalmár ${ }^{5}$ (D)
}

Received: 25 March 2019 / Accepted: 13 August 2019 / Published online: 2 September 2019

(C) The Author(s) 2019

\begin{abstract}
Objective The prevalence of xeroderma pigmentosum (XP) is quite low in Europe, which may result in a delay in determining the appropriate diagnosis. Furthermore, some subtypes of XP, including XPA, may manifest themselves with quite severe neurological symptoms in addition to the characteristic dermatological lesions. Accordingly, the aim of the current study is to highlight the predominant neurological aspects of XPA, as well as mild-to-moderate dermatological signs in a Hungarian family with 5 affected siblings. Case reports The symptoms of the Caucasian male proband started to develop at 13-14 years of age with predominantly cerebellar, hippocampal, and brainstem alterations. His elder sister and three younger brothers all presented similar, but less expressed neurological signs. The diagnostic work-up, including clinical exome sequencing, revealed 2 novel compound heterozygous mutations (p.Gln146_Tyr148delinsHis, p.Arg258TyrfsTer5) in the XPA gene. Surprisingly, only mild-to-moderate dermatological alterations were observed, and less severe characteristic ophthalmological and auditory signs were detected. Conclusions In summary, we present the first family with genetically confirmed XPA in the Central-Eastern region of Europe, clearly supporting the notion that disturbed function of the C-terminal region of the XPA protein contributes to the development of age-dependent neurologically predominant signs. This case series may help clinicians recognize this rare disorder.
\end{abstract}

Keywords Xeroderma pigmentosum group A $\cdot$ Neurological $\cdot$ Ataxia $\cdot$ Parkinsonism $\cdot$ Cognitive $\cdot$ Neuropathology

\section{Introduction}

Xeroderma pigmentosum (XP) is a rare autosomal recessively inherited condition with $100 \%$ penetrance [1] and only with a prevalence of 2.3 per million in Western Europe [2]. There are several distinct clinical subtypes of XP, including the variant form and those marked with letters $A-G$ based on the different genetic cause. The characteristic cutaneous signs are exaggerated

Electronic supplementary material The online version of this article (https://doi.org/10.1007/s10072-019-04044-6) contains supplementary material, which is available to authorized users.

Tibor Kalmár

kalmar.tibor@med.u-szeged.hu

1 Department of Neurology, Faculty of Medicine, Albert Szent-Györgyi Clinical Center, University of Szeged, Szeged, Hungary

2 Department of Dermatology and Allergology, Faculty of Medicine, Albert Szent-Györgyi Clinical Center, University of Szeged, Szeged, Hungary

3 Department of Pathology, Faculty of Medicine, Albert Szent-Györgyi Clinical Center, University of Szeged, Szeged, Hungary
4 Institute of Neurology, Medical University of Vienna, Vienna, Austria

5 Genetic Diagnostic Laboratory, Department of Pediatrics and Pediatric Health Center, University of Szeged, Szeged, Korányi fasor 14-15, Szeged H-6720, Hungary

6 Department of Ophthalmology, Faculty of Medicine, Albert Szent-Györgyi Clinical Center, University of Szeged, Szeged, Hungary

7 MTA-SZTE Neuroscience Research Group, University of Szeged, Szeged, Hungary

8 Interdisciplinary Excellence Center, University of Szeged, Szeged, Hungary 
sensitivity to sunlight, early development of freckle-like lentiginous pigmentation, and increased propensity for the formation of malignant skin tumors. The ocular alterations may involve conjunctival xerosis, corneal drying, and conjunctivitis. Neurological signs, such as intellectual disability, speech disturbance, sensorineural hearing loss, peripheral neuropathy, corticospinal alterations, and movement disorders with a predominant cerebellar ataxia resulting in severe walking disability, may also develop, especially in XPA [1,3]. The aim of the current study is to present a family with 5 affected siblings diagnosed with novel compound heterozygous mutations in the XPA gene in accordance with the CARE (CAse REport) guidelines [4]. Additionally, this report is unique in its detailed genetical, neuropathological, ophthalmological, and dermatopathological assessment in addition to the comprehensive delineation of clinical symptoms and signs. Accordingly, this case series may add to the known phenotypic spectrum of XPA.

\section{Case reports}

\section{Case histories, neurological, and related alterations}

The Caucasian male proband (II-2 in Fig. 1), who died at 39 years of age, was first admitted to our clinic for a diagnostic work-up of his unknown cognitive and movement disorder at 36 years of age. In addition to the presence of a slightly exaggerated sunburn reaction, his neurological symptoms started to develop at his 13-14 years of age. His speech became slurred and his cognitive functions deteriorated as well, resulting in progressive and severe learning disabilities. He completed 11 classes and later he worked in a twine factory until his 26 years of age, and then his disability led to retirement, and he became dependent on his parents. Repeated falls occurred with scarring and his swallowing functions and vision deteriorated as well.

Upon neurological examination, he presented signs of disturbed eye movements (exophoria, restricted eye movements in all directions with diplopia, gaze-evoked nystagmus), dysarthria, hypo-/areflexia, pathological reflexes, and decreased sense of vibration. Furthermore, movement disorder with dominating ataxia and parkinsonism (bilateral dysmetria, cerebellar predominant mixed limb ataxia more pronounced in the legs, truncal ataxia, severe postural instability, broadbased, ataxic gait, moderate, mainly left-sided bradykinesia and upper limb dystonia, mild rigidity on provocation, mild postural tremor, and occasional myoclonic jerks) was also detected (Supplementary Material 1.1 and 2). The myoclonic jerks were spontaneous, distal, and as they can be exacerbated by sensory stimuli, they were presumed to be cortical. The neuropsychological assessment revealed the signs of severe cognitive dysfunction confined to two functional neuroanatomical networks, the hippocampus-dependent and that related to the prefronto-cerebellar system, with similar degrees of impairment (Supplementary Material 1.2).

The brain MRI revealed pronounced generalized atrophy with slight predominance regarding the parieto-occipital and cerebellar structures (Supplementary Material 1.3). The degree of atrophy seemed to correlate with the severity of neurological signs.

Electroneurography demonstrated mixed sensorimotor lower limb predominant polyneuropathy.

Following his death caused by aspiration pneumonia resulting from dysphagia as a part of progressive neurological impairments, comprehensive post mortem neuropathological assessment was carried out. The most prominent alterations demonstrated by that were asymmetrical hippocampal sclerosis and Purkinje cell degeneration along with moderate loss of neurons in the substantia nigra and a scattered infiltration of CD8-positive T lymphocytes (for details, please see Supplementary Material 1.4 and 1.5).

Regarding the family history of the proband, the presence of similar, but less expressed, deterioration was identified in his sister (II-1, 3 years older than the probandSupplementary Materials 1.6 and 3), and his three brothers (II-3, 3 years younger than the proband-Supplementary Materials 1.7 and 4; II-4, 5 years younger than the proband-Supplementary Materials 1.8 and 5; II-5, 14 years
Fig. 1 Pedigree of the assessed family with mutation in the XPA gene

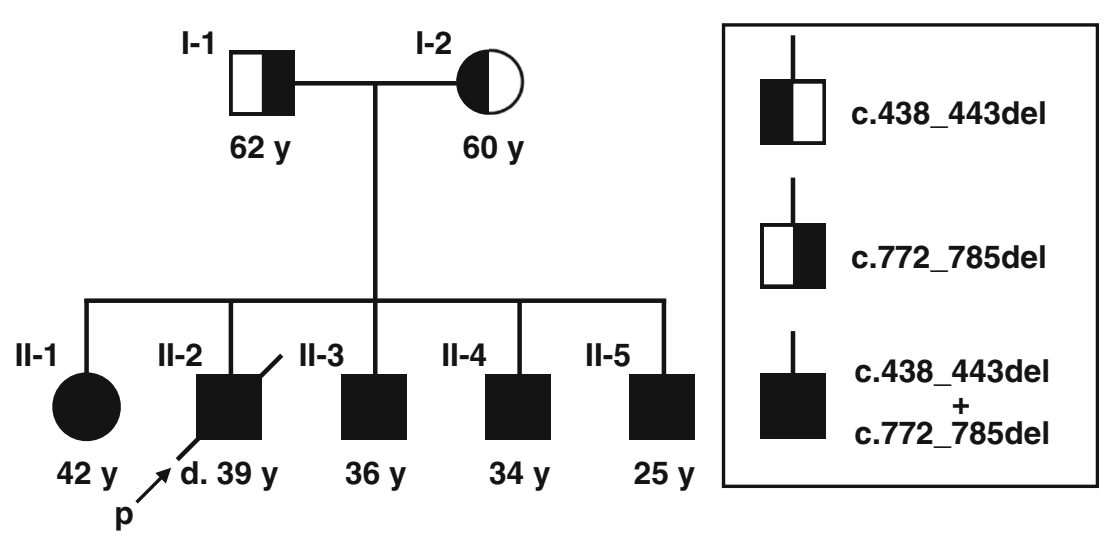


younger than the proband-Supplementary Materials 1.9 and 6), respectively (Fig. 1). Besides a mild light sensitivity in the father (I-1), there were no major relevant symptoms in the parents; the demonstrated slight cognitive impairment may have other explanations unrelated to XPA (Supplementary Material 1.2).

\section{Dermatological and dermatopathological assessments}

Dermatological alterations generally include mild-to-moderate solar damage on sun-exposed areas with the presence of hyperpigmentation in the basal keratinocytes and solid and infiltrative basal cell carcinomas (Supplementary Material 1.10).

The results of the neurological and dermatological examinations enabled staging of all 5 siblings according to the Japanese Dermatological Association's guideline (Table 1; [3]).

\section{Ophthalmological assessment}

Ophthalmological examinations demonstrated exophoria and weakness of convergence with gaze-dependent diplopia in patients II-1 and II-2. Pathological alterations of the vitreo-macular interface, i.e., thickened internal limiting membrane (ILM) with ruffled inner surface in the macular area of the retina, were diagnosed in patients II-2, II-3, and II-4. This sign can be considered as a gliotic proliferation of the inner surface of the ILM, although epiretinal membrane formation could not be detected.

\section{Diagnostic challenges and genomic studies}

The major diagnostic challenges were that besides the severe neurological symptoms and signs, the dermatological alterations were not so prominent to be easily recognized by a neurologist.

Variants obtained by exome sequencing (for details, please see Supplementary Material 1.11) were filtered based on severity and frequency against public variant databases including dbSNP, ClinVar, ExAC, EVS, GnomAD, and an in-house clinical exome database of 300 unrelated Hungarian samples.

For the two new XPA variants (NM_000380.3:c.438_443delAGAATA; NP_000371.1:p.Gln146_Tyr148delinsHis and NM_000380.3:c.772_785delCGTAAGACTTGTAC; NP_000371.1:p.Arg258TyrfsTer5), ClinVar accessions VCV000523609.1 and VCV000523609.1 were assigned, respectively. The minor allele frequency (MAF) for XPA c.438_443delAGAATA variant is unknown since it is not listed in either the gnomAD (https://gnomad.broadinstitute.org/), ExAc (http://exac.broadinstitute.org/), or in the EVS (https://evs.gs. washington.edu/EVS/) databases. The MAF for XPA c.772 785 delCGTAAGACTTGTAC variant is 0.0000814 (23/ 282538 allele) according to the gnomAD (https://gnomad. broadinstitute.org/) database, which is within the range for a pathogenic recessive allele. Based on the American College of Medical Genetics and Genomics variant interpretation guidelines [5], the first variant was classified as likely pathogenic, whereas the second as pathogenic. The conservation of the region of XPA protein affected by the in-frame mutation is demonstrated by Supplementary Material 1.12.

\section{Discussion}

Regarding genotype-phenotype correlation, mutations affecting exons 2, 3 and introns 1, 3 in the XPA gene are almost
Table 1 The staging of XPA patients according to the classification of the severity of XP proposed by the Japanese Dermatological Association's guidelines [3]

\begin{tabular}{|c|c|c|c|c|c|}
\hline & Patient II-1 & Patient II-2 & Patient II-3 & Patient II-4 & Patient II-5 \\
\hline \multicolumn{6}{|l|}{ Cutaneous symptom (D) score } \\
\hline Exaggerated sunburn & 3 & 3 & 3 & 3 & 3 \\
\hline Freckle-like eruption & 3 & 3 & 1 & 2 & 0 \\
\hline Skin cancer & 3 & 2 & 0 & 0 & 0 \\
\hline Severity of cutaneous symptoms & D3 & D3 & D2 & D2 & D2 \\
\hline \multicolumn{6}{|l|}{ Extracutaneous symptom $(\mathrm{N})$ score } \\
\hline Hearing ability & 1 & N.A. & 1 & 0 & 0 \\
\hline Movement & 2 & 3 & 2 & 2 & 2 \\
\hline Intellectual functions & 2 & 3 & 2 & 2 & 2 \\
\hline Swallowing and respiratory function & 0 & 3 & 0 & 0 & 0 \\
\hline $\begin{array}{l}\text { Severity of extracutaneous } \\
\text { symptoms }\end{array}$ & $\mathrm{N} 3$ & N3 & $\mathrm{N} 3$ & $\mathrm{~N} 2$ & $\mathrm{~N} 2$ \\
\hline \multicolumn{6}{|c|}{ Classification of XP depending on the severity } \\
\hline Stage & 4 & 4 & 4 & 3 & 3 \\
\hline
\end{tabular}

$X P$, xeroderma pigmentosum; N.A., not available 
always accompanied by severe characteristic clinical signs, whereas mutation sites approaching the $\mathrm{C}$-terminal of the corresponding XPA protein may be considered as hypomorphic, i.e., they can be characterized by less severe neurological and especially, dermatological phenotypes [6, 7] (the clinical and demographic characteristics of all the published mutations in the XPA gene were collected and outlined in Supplementary Material 1.13). Indeed, cutaneous signs may be absent as well [8]. In addition to the mutation site, the clinical phenotype may be influenced by the age of the patient and, for dermatological and ophthalmological alterations, by sun exposure as well, even within the same family [9].

This genotype-phenotype correlation was confirmed by the current study as well, i.e., only mild-to-moderate dermatological and no prominent ophthalmological and audiological, but severe neurological signs evolved with age.

Despite the hypomorphic character of the reported variants, the likely pathogenic feature of the in-frame mutation in exon 4 (p.Gln146_Tyr148delinsHis) is supported by that it affects a conserved region of the protein (presented in Supplementary Material 1.12) including p.Glu147 and p.Tyr148, the deletion of which results in the pathologically reduced binding of XPA protein to replication protein A [10]. The second one in exon 6 (p.Arg258TyrfsTer5) may be considered pathogenic as it eventuates a premature stop codon. In addition to its frameshift character, the pathogenicity of this variant may be further supported by a later unrelated report of it in a patient with XPA (https://www.ncbi.nlm.nih.gov/ clinvar/variation/523608/).

Furthermore, in addition to the presentation of a very detailed phenotypic description from a neurological point of view, the present study yields several novelties: (1) 5 out of 5 affected siblings within a single family has not been reported before, and this study presents the first cases of XPA in the Central-Eastern region of Europe. (2) Likely pathogenic novel in-frame and pathogenic novel frameshift mutations in trans position are presented. (3) A previously unreported scattered infiltration of $\mathrm{CD} 8+\mathrm{T}$ lymphocytes was detected in the proband with XPA, without a manifest corresponding dermatological alteration. In light of the fact that in common neurodegenerative diseases with accumulation of proteins, CD8+ cytotoxic $\mathrm{T}$ cells are not considered as hallmark lesions; this finding is unexpected and may be part of an immunological activation following an insult from a currently uncharacterized agent or process. However, the significance of this observation could not be clarified in the present study and merits further observations on similar cases.

In conclusion, the presentation of this unusual neurological predominant phenotype of XPA may help clinicians recognize this rare disorder.
Acknowledgements Open access funding provided by University of Szeged (SZTE).

Funding information This study was supported by the GINOP-2.3.2-152 grant (TK, ZM), by the GINOP-2.3.2-15-2016-00034 and 20391-3/ 2018/FEKUSTRAT grants (LV), by the OTKA125509 and GINOP2.3.2-15-2016-00020 grants (IBN), and by the National Brain Research Program 2017-1.2.1-NKP-2017-00002_VI/4 (PK, LV) provided by The National Research, Development and Innovation Office (Hungary). The state funders played no role in the study design, data collection or analysis, decision to publish, or in the preparation of the manuscript. DZ and IBN were supported by the János Bolyai Research Scholarship of the Hungarian Academy of Sciences.

\section{Compliance with ethical standards}

Conflict of interest The authors declare that they have no conflict of interest.

Ethical approval Written informed consent was obtained from all individual participants included in the study. Additional informed consent was obtained from all individual participants for whom identifying information is included in this article (Regional Human Biomedical Research Ethics Committee of the University of Szeged registration numbers are 150/2014. and 44/2016., respectively). All procedures performed in studies involving human participants were in accordance with the ethical standards of the Regional Human Biomedical Research Ethics Committee of the University of Szeged and with the 1964 Helsinki declaration and its later amendments or comparable ethical standards.

Open Access This article is distributed under the terms of the Creative Commons Attribution 4.0 International License (http:// creativecommons.org/licenses/by/4.0/), which permits unrestricted use, distribution, and reproduction in any medium, provided you give appropriate credit to the original author(s) and the source, provide a link to the Creative Commons license, and indicate if changes were made.

\section{References}

1. Black JO (2016) Xeroderma pigmentosum. Head Neck Pathol 10: 139-144. https://doi.org/10.1007/s12105-016-0707-8

2. Kraemer KH, Lee MM, Scotto J (1987) Xeroderma pigmentosum. Cutaneous, ocular, and neurologic abnormalities in 830 published cases. Arch Dermatol 123:241-250

3. Moriwaki S, Kanda F, Hayashi M, Yamashita D, Sakai Y, Nishigori $\mathrm{C}$, Xeroderma pigmentosum clinical practice guidelines revision committee (2017) Xeroderma pigmentosum clinical practice guidelines. J Dermatol 44:1087-1096. https://doi.org/10.1111/13468138.13907

4. Gagnier JJ, Kienle G, Altman DG, Moher D, Sox H, Riley D, CARE Group* (2013) The CARE guidelines: consensus-based clinical case reporting guideline development. Glob Adv Health Med 2:38-43. https://doi.org/10.7453/gahmj.2013.008

5. Richards S, Aziz N, Bale S et al (2015) Standards and guidelines for the interpretation of sequence variants: a joint consensus recommendation of the American College of Medical Genetics and Genomics and the Association for Molecular Pathology. Genet Med Off J Am Coll Med Genet 17:405-424. https://doi.org/10. 1038/gim.2015.30

6. Takahashi Y, Endo Y, Sugiyama Y, Inoue S, Iijima M, Tomita Y, Kuru S, Takigawa M, Moriwaki S (2010) XPA gene mutations 
resulting in subtle truncation of protein in xeroderma pigmentosum group A patients with mild skin symptoms. J Invest Dermatol 130: 2481-2488. https://doi.org/10.1038/jid.2010.137

7. Messaoud O, Rekaya MB, Ouragini H et al (2012) Severe phenotypes in two Tunisian families with novel XPA mutations: evidence for a correlation between mutation location and disease severity. Arch Dermatol Res 304:171-176. https://doi.org/10.1007/s00403011-1190-4

8. Messaoud O, Ben Rekaya M, Kefi R, Chebel S, BoughammouraBouatay A, Bel Hadj Ali H, Gouider-Khouja N, Zili J, Frih-Ayed M, Mokhtar I, Abdelhak S, Zghal M (2010) Identification of a primarily neurological phenotypic expression of xeroderma pigmentosum complementation group $\mathrm{A}$ in a Tunisian family. $\mathrm{Br}$
J Dermatol 162:883-886. https://doi.org/10.1111/j.1365-2133. 2010.09646.x

9. Kondoh M, Ueda M, Ichihashi M (1995) Correlation of the clinical manifestations and gene mutations of Japanese xeroderma pigmentosum group A patients. Br J Dermatol 133:579-585

10. Feltes BC, Bonatto D (2015) Overview of xeroderma pigmentosum proteins architecture, mutations and post-translational modifications. Mutat Res Rev Mutat Res 763:306-320. https://doi.org/10. 1016/j.mrrev.2014.12.002

Publisher's note Springer Nature remains neutral with regard to jurisdictional claims in published maps and institutional affiliations. 